\title{
Essential Thrombocythemia
}

\section{SUSAN S GRAHAM}

\section{LEARNING OBJECIVES}

1. Identify the mutations associated with essential thrombocythemia (ET).

2. Describe the clinical presentation of patients with essential thrombocythemia.

3. Recall the $2008 \mathrm{WHO}$ criteria for diagnosis of essential thrombocythemia.

4. Differentiate essential thrombocythemia from other myeloproliferative neoplasms and reactive thrombocytosis.

5. Discuss the expected clinical course of patients with essential thrombocythemia.

Clin Lab Sci 2011;24(3):187

Susan S Graham, MS, MT (ASCP)SH $H^{C M}$, College of Health Professions, SUNY Upstate Medical University, Syracuse, NY

Address for Correspondence: Susan S Graham, MS, MT (ASCP)SH ${ }^{C M}$, Dept. of Clinical Laboratory Science, Assistant Dean, College of Health Professions, SUNY Upstate Medical University, 750 East Adams Street, Syracuse, NY 13210,315-464-5334,grahams@upstate. edu

\section{INTRODUCTION}

Essential thrombocythemia (ET) is a clonal stem cell disorder characterized by sustained thrombocytosis > $450 \times 10^{9} / \mathrm{L}$ in the peripheral blood, increased numbers of large, mature megakaryocytes in the bone marrow, and clinically by episodes of thrombosis or hemorrhage. ${ }^{1}$ Initially described in 1934, ET was recognized as a primary thrombocythemia and ultimately grouped with other myeloproliferative neoplasms with pancellular hyperproliferation of bone marrow elements, increased cellular presence in the peripheral blood, and organomegaly. ${ }^{2}$
The discovery of the Philadelphia chromosome and its subsequent association with the $B C R / A B L 1$ mutation led the way to the grouping of ET with the other $B C R / A B L$-negative myeloproliferative neoplasms, polycythemia vera (PV) and primary myelofibrosis (PMF). It was later found that these disorders shared the Janus kinase 2 (JAK2 V617F) mutation. This mutation was found in $90 \%$ of patients with PV, and $50 \%$ of patients with either ET or PMF. ${ }^{3}$

This article explores the pathology and laboratory presentation of ET along with the diagnosis and clinical course of the disease.

\section{Pathology}

The etiology of ET is unknown. Studies suggest that the JAK2 V617F mutation is associated with cytokineindependent proliferation of cytokine-dependent cell lines. ${ }^{4}$ While this would be consistent with the hyperproliferation of the megakaryocytic cell line, it does not entirely explain the abnormality, given that approximately half of the patients with ET are $J A K 2$ V617F negative. JAK2 V617F activates a tyrosine kinase of the erythropoietin and thrombopoietin receptors that transform hematopoietic precursors. Mutations in $M P L$ and TET2 oncogenes have also been identified in some patients with ET although they are not specific to this disorder.

\section{Clinical Presentation}

Essential thrombocythemia is characterized by an insidious onset, with approximately $50 \%$ of patients being asymptomatic at diagnosis. An isolated, unexplained thrombocytosis may be discovered as part of a routine physical or elective procedure. The remaining $50 \%$ of patients may have evidence of either thrombosis or hemorrhage. ${ }^{1}$ Arterial thrombosis is most common, but venous occlusions have been reported as well. If the disorder manifests itself with hemorrhage, the bleeding predominantly involves mucosal surfaces. 


\section{FOCUS: UPDATE ON MYELOPROLIFERATIVE NEOPLASMS}

Splenomegaly may be present due to platelet sequestration and not extramedullary hematopoiesis. Most cases present in the $5^{\text {th }}$ or $6^{\text {th }}$ decade and are equally distributed between males and females. ${ }^{1}$ Another, smaller cohort of patients may be seen at approximately 30 years of age and is primarily female.

\section{Laboratory Findings \\ Peripheral Blood}

Marked thrombocytosis is the most striking finding in the peripheral blood. Platelet size is variable, and may include bizarre forms and shapes. ${ }^{1}$ The WBC count is normal to slightly increased and the WBC differential is normal. RBC morphology is normocytic and normochromic unless complicated by iron deficiency. This complication is most frequently seen when symptoms include recurrent hemorrhage, depleting the iron stores. It is important to note that there is no evidence of teardrop cells or leukoerythroblastosis as these would be more indicative of PMF.

\section{Bone Marrow}

The bone marrow appears normocellular or slightly hypercellular. ${ }^{1}$ The most significant finding is the presence of increased megakaryocytes. Careful consideration of the morphology gives important clues to aid in differentiation from other causes of thrombocytosis. Large to giant megakaryocytes with abundant cytoplasm and hyperlobulated nuclei are characteristic of ET, as opposed to the dwarf forms more commonly seen in CML. ${ }^{1}$ The appearance of bizarre, highly atypical megakaryocytes would be a finding more consistent with myelofibrosis.

$\mathrm{RBC}$ precursors are typically normal in number and appearance, although an increase in $\mathrm{RBC}$ precursors may be seen in cases involving significant bleeding. There is no increase in myeloblasts or myelodysplastic features. A combined increase in RBC and WBC elements would be more suggestive of PV. Additionally, reticulin fibrosis is not seen.

\section{Genetics}

The $J A K 2$ V617F mutation is present in approximately $50 \%$ of cases and the $M P L$ mutation is reported in 1$4 \%$ of cases. ${ }^{1,4}$ Additional cytogenetic abnormalities found in patients with ET include +8 , abnormalities of
$9 \mathrm{q}$, and $\operatorname{del}(5 \mathrm{q}) .{ }^{1}$ These are found infrequently and are not specific for the disorder.

\section{Differential Diagnosis}

Essential thrombocythemia has long been a diagnosis of exclusion. According to the $\mathrm{WHO}^{1}$, diagnosis requires a persistent platelet count $\geq 450 \times 10^{9} / \mathrm{L}$; examination of a bone marrow specimen to demonstrate the characteristic abnormalities of the megakaryocytic lineage; and exclusion of reactive thrombocytosis, polycythemia vera, primary myelofibrosis, chronic myelogenous leukemia, myelodysplastic syndrome or other myeloid neoplasm.

Polycythemia vera is distinguished from ET by a decreased serum erythropoietin level and elevation of the hemoglobin concentration. ${ }^{5}$ Primary myelofibrosis, like ET, shows evidence of megakaryocytic proliferation with abnormal forms in the bone marrow, but found with the presence of reticulin or collagen fibrosis. ${ }^{5}$ The absence of $B C R-A B L 1$ or dyserythropoiesis and dysgranulopoiesis effectively rules out CML and PMF, respectively.

Reactive thrombocytosis is the most common type of thrombocytosis. One study demonstrated that $82 \%$ of patients with platelet counts > $1000 \times 10^{9} / \mathrm{L} \mathrm{had}$ secondary thrombocytosis while only $14 \%$ has a myeloproliferative neoplasm. ${ }^{6}$ A subsequent study of 535 patients with counts $>1000 \times 10^{9} / \mathrm{L}$ ) showed the etiology to be secondary thrombocytosis in $66.6 \%$ of cases. $^{7}$ The remainder were clonal in origin. Platelet production in reactive thrombocytosis is increased by an elevation of thrombopoietin secondary to inflammation, infection, neoplastic conditions or stress. ${ }^{8}$ Causes of secondary (reactive) thrombocytosis include acute blood loss, iron deficiency, hemolytic anemia, chronic or acute inflammation or infection, and drug reactions. The presence of giant platelets in the peripheral blood supports the diagnosis of ET. None of the genetic mutations $(J A K 2, M P L)$ is found in reactive thrombocytosis.

\section{Diagnosis}

The best approach in a patient with an unexplained, persistent thrombocytosis is to test for the JAK2 V617F mutation. If positive, rule out $\mathrm{PV}$ by demonstrating 


\section{FOCUS: UPDATE ON MYELOPROLIFERATIVE NEOPLASMS}

normal iron stores and RBC mass. Then, exclude other causes by demonstrating the absence of an enlarged spleen $(>5 \mathrm{~cm})$, underlying systemic disease, and evidence of leukoerythroblastic or dysplastic features on the blood film. If the sample is negative for the $J A K 2$ V617F mutation, or if there is evidence of other causes, proceed with a bone marrow aspiration and biopsy and apply the diagnostic criteria outlined in the $2008 \mathrm{WHO}$ classification (Table 1). ${ }^{9}$

Table 1. WHO Criteria for Diagnosis of Essential

Thrombocythemia ${ }^{1}$

All four criteria must be met:

1. Sustained platelet count $\geq 450 \times 10^{9} / \mathrm{L}$

2. Bone marrow specimen showing proliferation mainly of the megakaryocytic lineage with increased numbers of enlarged, mature megakaryocytes. No significant increase or left-shift of neutrophil granulopoiesis or erythropoiesis.

3. Not meeting WHO criteria for polycythemia vera, primary myelofibrosis, BCR/ABL1 positive chronic myelogenous leukemia or myelodysplastic syndrome or other myeloid neoplasm.

4. Demonstration of JAK2 V617F or other clonal marker, or in the absence of JAK2 V617F, no evidence for reactive thrombocytosis.

\section{Clinical Course}

Essential thrombocythemia is a chronic myeloproliferative neoplasm with a characteristic long-term survival and near normal life expectancy. Patients experience long asymptomatic intervals with infrequent thrombotic or hemorrhagic complications. Decisions regarding treatment are usually based on the presence of cardiovascular risk factors, such as hypertension, diabetes, smoking, hypercholesterolemia and obesity. To prevent thrombosis, aspirin is recommended for all ET patients. ${ }^{9}$
Therapy to reduce the platelet count is considered in patients older than 60 years of age at diagnosis or those having had a previous thrombosis. The platelet count alone does not correlate with thrombotic risk. Indeed, a high platelet count may be associated with hemorrhagic risk. In such cases, hydroxycarbamide (hydroxyurea) is the cytoreductive agent used. ${ }^{?}$

Current research is focused on developing JAK2 inhibitors that would inhibit cytokine-independent proliferation of hematopoietic stem cells. ${ }^{3}$ Most compounds are undergoing pre-clinical and clinical trials to test the effect of JAK2 inhibition on other cells requiring $J A K 2$ signaling in an effort to avoid detrimental effects such as lack of erythropoiesis. In the absence of demonstrated toxicity to other cells lines, this approach is quite promising.

\section{REFERENCES}

1. Thiele J, Kvasnicka HM, Orazi A, et al. Essential Thrombocythaemia In: Swerdlow SH, Campo E, Harris NL, et al. WHO Classification of Tumours of Haematopoietic and Lymphoid Tissues. Lyon: IARC Press. 2008:48-50.

2. Tefferi A. The history of myeloproliferative disorders: before and after Dameshek. Leukemia. 2008;22:3-13.

3. Levine RL, Pardanani A, Tefferi A, Gilliland DG. Role of $J A K 2$ in the pathogenesis and therapy of myeloproliferative disorders. Nat Rev Cancer. 2007;9:673-83.

4. Beer PA, Green AR. Pathogenesis and management of essential thrombocythemia. Hematology Am Soc Hematol Educ program. 2009:621-8.

5. Tefferi A, Vainchenker W. Myeloproliferative neoplasms: molecular pathophysiology, essential clinical understanding, and treatment strategies. J Clin Oncol. 2011;28:1-10.

6. Buss DH, Cashell AW, O'Connor ML, et al. Occurrence, etiology, and clinical significance of extreme thrombocytosis: a study of 280 cases. Am J Med. 1994;96:247-53.

7. Wiwanilit V. Extreme thrombocytosis: what are the etiologies? Clin Appl Thromb Hemost. 2006;12:85-7.

8. Schafer AI. Thrombocytosis. N Engl J Med. 2004;350:1211-9.

9. Beer PA, Erber WN, Campbell PJ, Green AR. How I treat essential thrombocythemia. Blood. 2011;117:1472-82. 